\title{
ARRAY ANALYSIS OF SEISMIC SIGNALS
}

\author{
Peter Goldstein and Ralph J. Archuleta \\ Department of Geological Sciences, University of California, Santa Barbara CA 93106
}

\begin{abstract}
In this paper we present a method for analyzing seismic signals recorded at an array of seismometers. The method is based on the multiple signal characterization (MUSIC) method. Four important features of this approach are: (1) it has the ability to resolve multiple closely spaced sources, (2) it works with both stationary, and nonstationary signals, (3) it is most sensitive to the strongest sources, and (4) it provides a geometric interpretation of the solution to the direction finding problem. We have developed a numerical algorithm that extends the MUSIC method to nonstationary and correlated signals. We present results using this algorithm to measure directions of arrival and amplitudes of multiple plane waves in a homogeneous medium.
\end{abstract}

\section{Introduction}

Seismic arrays have proved useful in a variety of problems, for example, discrimination between nuclear explosions and earthquakes [e.g., Ringdal and Husebye, 1982], determination of earth structure from crust to core [Capon, 1974, Filson, 1975, Aki et al., 1976], determination of the strike and dip of the Moho [Haskov and Kanasewich, 1978], measurements of near source ground accelerations [McLaughin et al., 1983], and observations of earthquake rupture propagation [Spudich and Cranswick, 1984, Abrahamson, 1985]. The pioneering work of Capon [1967, 1969] has served as the basis for most of the analysis of seismic data collected using specialized arrays, excepting techniques developed for seismic reflection. Although Capon's [1969] high resolution maximum likelihood technique has been successfully applied in certain cases, developments in array signal processing in fields outside of seismology during the past decade have produced several techniques [see Kay and Marple, 1981, or Haykin, 1985, for reviews] with resolving power superior to Capon's high resolution method. In particular, Schmidt's [1981, 1986] multiple signal characterization (MUSIC) method has features, described below, that make it extremely well suited for array analysis of seismic data.

We have started to develop a numerical algorithm based on MUSIC specifically for array analysis of seismic signals. In preliminary tests of the method we have been able to demonstrate its superior resolution over Capon's method. Capon's method and other modifications assume that the observed time series is stationary, and its constituent signals are uncorrelated. While these assumptions may apply to certain parts of a seis-

Copyright 1987 by the American Geophysical Union.

Paper number 6L 7069 .

0094-8276/87/006L-7069\$03.00 mogram, e.g., the coda, they certainly do not apply to transient signals, e.g., body waves, or to signals that arrive simultaneously from different directions (multipathing). We have adapted MUSIC to measure both nonstationary and stationary signals.

\section{Theory and Method}

In the following we illustrate Schmidt's [1981,1986] signal subspace approach using a special case of plane waves propagating in a homogeneous medium. For simplicity in the following discussion we consider a single component of motion although MUSIC allows for multiple components.

Suppose that a set of $q(<N)$ plane waves with angular frequency $\omega$ are incident on an array of $N$ sensors in a homogeneous medium . The narrowband signal received at station $\vec{x}_{i}$ at time $t$ is given by

$$
\psi\left(\vec{x}_{i}, t\right)=\sum_{m=1}^{q} A_{m} e^{i\left(\vec{k}_{m} \cdot \vec{x}_{i}-\omega t+\phi_{m}(t)\right)}+\eta\left(\vec{x}_{i}, t\right),
$$

where $\eta\left(\vec{x}_{i}, t\right)$ is the noise, $\vec{k}_{m}$ is the wavevector of the $m$ th signal, and $\phi_{m}(t)$ is the phase of the $m$ th signal. A stationary signal exists when $\phi_{m}(t)$ is a random function of time. A correlated stationary signal exists when any $\phi_{i}(t)$ and $\phi_{j}(t)$ are random but correlated with each other. A nonstationary signal exists when $\phi_{m}(t)$ is not a random function of time. In the following we assume that the noise $\eta\left(\vec{x}_{i}, t\right)$ is random and uncorrelated with the signal. The covariance of the signal received at stations $i$ and $j$ is defined as

$$
R_{i j}=\left\langle\psi\left(\vec{x}_{i}, t\right) \psi^{\dagger}\left(\vec{x}_{j}, t\right)\right\rangle_{t}
$$

where \langle\rangle$_{t}$ means time average, and $\dagger$ indicates Hermitian conjugate. $R_{i j}$ is a measure of the correlation between signals received at $\vec{x}_{i}$ and signals received at $\vec{x}_{j}$. When the $q$ signals are stationary, the elements of the covariance matrix are

$$
R_{i j}=\sum_{m=1}^{q}\left|A_{m}\right|^{2} e^{i \vec{k}_{m} \cdot\left(\vec{x}_{i}-\vec{x}_{j}\right)}+\sigma^{2} \delta_{i j},
$$

where $\sigma^{2}$ is the noise intensity. Thus, the observed covariances are a linear superposition of individual signal covariances plus some measure of noise. Defining the observed signal vector as

$$
\vec{\Psi}(t)=\left[\psi\left(\vec{x}_{1}, t\right), \psi\left(\vec{x}_{2}, t\right), \ldots, \psi\left(\vec{x}_{N}, t\right)\right]^{T},
$$

where $T$ means transpose, the covariance matrix is

$$
\mathbf{R}=\left\langle\vec{\Psi}(t) \otimes \vec{\Psi}^{\dagger}(t)\right\rangle_{t},
$$

where $\otimes$ indicates the outer product of two vectors. The signal vector can be written as 


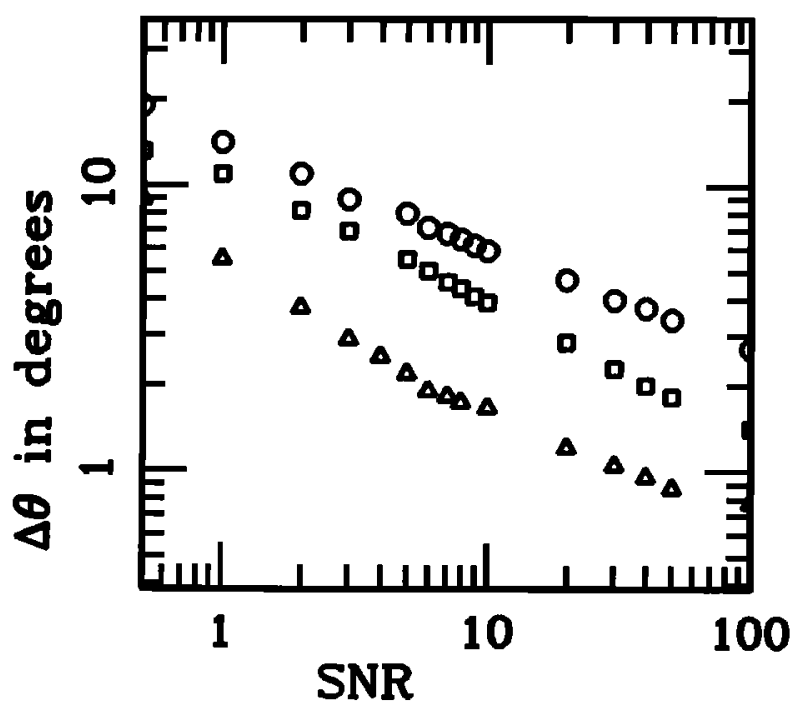

Fig. 1. Minimum angular resolution between two signals vs. signal to noise amplitude ratio (SNR). For two stationary signals MUSIC's results are shown as $\triangle$ 's and Capon's by $\square$ 's. For nonstationary signals MUSIC's results are shown as $O$ 's. For stationary signals the angular resolution of MUSIC is approximately $2 \frac{1}{2}$ times better than Capon's resolution. For nonstationary signals MUSIC's resolution is decreased by a factor of approximately $3 \frac{1}{2}$ from the stationary case.

$$
\vec{\Psi}(t)=\sum_{m=1}^{q} A_{m} \vec{u}\left(\vec{k}_{m}\right) e^{-i\left(\omega t+\phi_{m}(t)\right)},
$$

where the directional dependence is contained in the signal direction vectors

$$
\vec{u}\left(\vec{k}_{m}\right)=\left[e^{i \vec{k}_{m} \cdot \vec{x}_{1}}, \ldots, e^{i \vec{k}_{m} \cdot \vec{x}_{N}}\right]^{T}
$$

The covariance matrix can then be written as

$$
\mathbf{R}=\sum_{m=1}^{q}\left|A_{m}\right|^{2} \vec{u}\left(\vec{k}_{m}\right) \otimes \vec{u}^{\dagger}\left(\vec{k}_{m}\right)+\sigma^{2} \mathbf{I},
$$

or

where

$$
\mathbf{R}=\mathbf{U S U}^{\dagger}+\boldsymbol{\sigma}^{2} \mathbf{I}
$$

$$
\begin{gathered}
\mathrm{U}=\left(\begin{array}{ccccc}
\vec{u}\left(\vec{k}_{1}\right) & \vec{u}\left(\vec{k}_{2}\right) & \ldots & \vec{u}\left(\vec{k}_{q}\right) \\
\downarrow & \downarrow & \ldots & \downarrow
\end{array}\right) \\
\mathbf{S}=\left(\begin{array}{cccccc}
\left|A_{1}\right|^{2} & 0 & \ldots & \ldots & \ldots & 0 \\
0 & \ddots & \ldots & \ldots & \ldots & \vdots \\
\vdots & \vdots & \left|A_{q}\right|^{2} & \ldots & \ldots & \vdots \\
\vdots & \vdots & \vdots & 0 & \ldots & \vdots \\
\vdots & \vdots & \vdots & \vdots & \ddots & \vdots \\
0 & \ldots & \ldots & \ldots & \ldots & 0
\end{array}\right),
\end{gathered}
$$

and $I$ is the identity matrix. In this approach we find the $q$ signal direction vectors $\vec{u}\left(\vec{k}_{m}\right)$ and then invert for their associated intensities $\left|A_{m}\right|^{2}$.

When $q$, less than $N$, plane waves propagate across an $\mathrm{N}$ station array, the covariance matrix of the signals USU ${ }^{\dagger}$ has rank $q$ and is nonnegative definite. In this case it is easy to show that the minimum eigenvalue of
$\mathbf{R}$ is $\sigma^{2}$ with multiplicity $N-q$. From this it follows that the number of signals can be determined from the number of large eigenvalues, i.e. those greater than $\sigma^{2}$. It also follows that the $N-q$ eigenvectors of $\mathbf{R}, \vec{e}_{i}$, associated with the minimum eigenvalue are orthogonal to the $q$ spatial signal vectors $\vec{u}\left(\vec{k}_{m}\right)$, i.e.,

$$
\vec{e}_{i} \cdot \vec{u}\left(\vec{k}_{j}\right)=0 ; \quad i=q+1, \ldots, N, \quad j=1, \ldots, q .
$$

Define $\mathrm{E}_{\mathrm{g}}$ as the $N \times q$ matrix whose columns are the $q$ eigenvectors of $\mathbf{R}$ associated with the $q$ large eigenvalues, $\Lambda_{\mathbf{g}}$ the $q \times q$ diagonal matrix whose diagonal elements are the large eigenvalues of $\mathbf{R}, \mathrm{E}_{\mathrm{n}}$ the $N \times N-q$ matrix whose columns are the eigenvectors of $\mathbf{R}$ associated with the $N-q$ small eigenvalues, and $\boldsymbol{\Lambda}_{\mathbf{n}}$ the $N-q \times N-q$ diagonal matrix whose diagonal elements are the minimum eigenvalues of $\mathbf{R}$. The covariance matrix can be written in terms of its eigenstructure as

$$
\mathbf{R}=\mathbf{E}_{\mathbf{g}} \mathbf{\Lambda}_{\mathbf{B}} \mathbf{E}_{\mathbf{g}}^{\dagger}+\mathbf{E}_{\mathbf{n}} \mathbf{\Lambda}_{\mathbf{n}} \mathbf{E}_{\mathbf{n}}^{\dagger}
$$

where $\mathbf{E}_{\mathrm{g}} \boldsymbol{\Lambda}_{\mathbf{g}} \mathbf{E}_{\mathbf{8}}^{\dagger}$ represents the signals contribution to the covariances and $\mathbf{E}_{\mathbf{n}} \mathbf{\Lambda}_{\mathbf{n}} \mathbf{E}_{\mathbf{n}}^{\dagger}$ represents the noise. The essence of the MUSIC method is to find the $q$ signals which give a best fit to the signal covariances.

With this in mind, define the array manifold as the set of vectors $\vec{a}(\vec{k})$ which correspond to the spatial dependence of plane wave vectors

$$
\vec{a}(\vec{k})=\left[e^{i \vec{k} \cdot \vec{x}_{1}}, e^{i \vec{k} \cdot \vec{x}_{2}}, \ldots, e^{i \vec{k} \cdot \vec{x}_{N}}\right]
$$

where $\vec{k}$ can have any value consistent with $|\vec{k}|=\omega / \beta$, where $\beta$ is the medium velocity. In general, the set of array manifold vectors can be computed for any given array configuration provided that the medium's velocity structure is known. It is also possible to determine $\vec{a}(\vec{k})$ empirically.

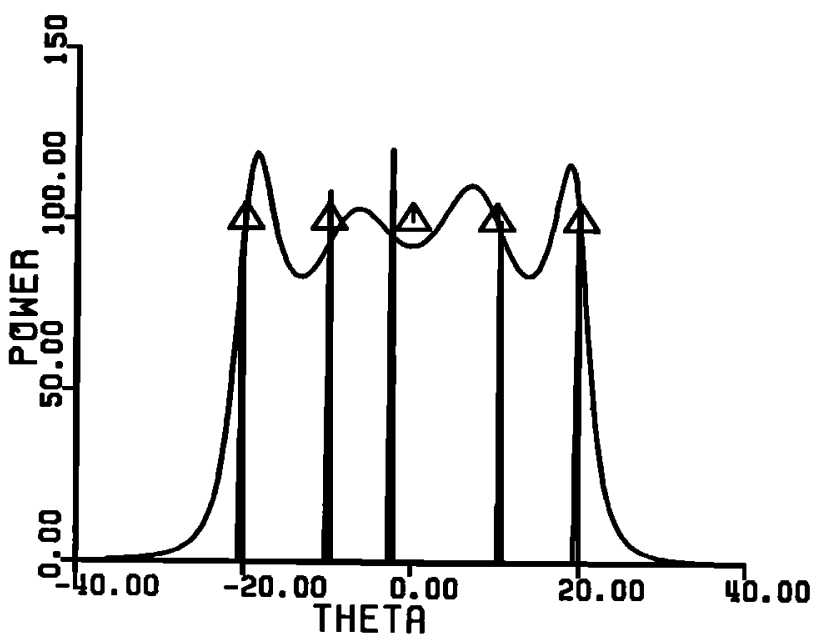

Fig. 2. Comparison of power spectra of five stationary signals at $0^{\circ}, \pm 10^{\circ}$, and $\pm 20^{\circ}$ with $\mathrm{SNR}=10.0$. Vertical lines are the MUSIC power estimates; the smooth curve is the power estimated by Capon's method. $\triangle$ 's correspond to input signals. 


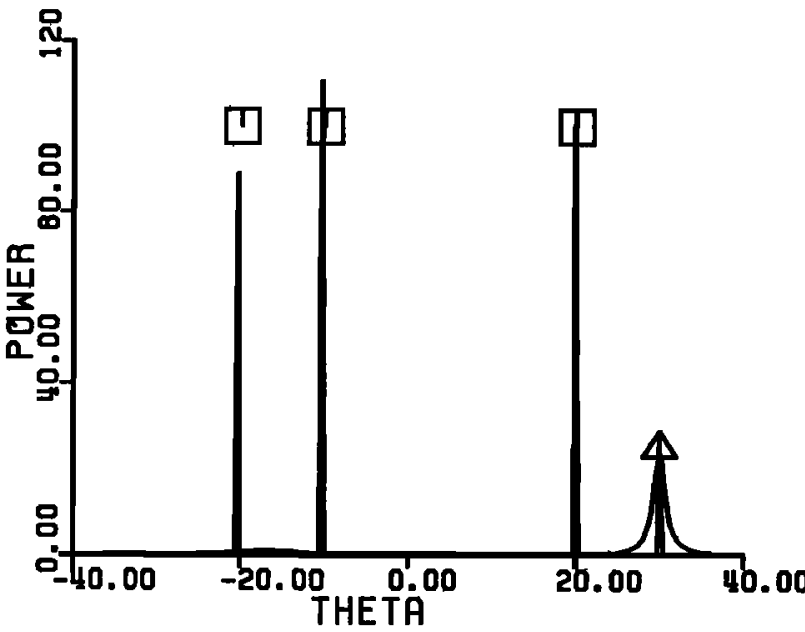

Fig.3. Comparison of power spectra for three nonstationary signals and one stationary signal. Open $\square$ 's correspond to input nonstationary signals; $\Delta$ 's input stationary signals. MUSIC acurately locates all four signals. Capon's method accurately locates only the stationary signal.

To determine the signal direction vectors $\vec{u}\left(\vec{k}_{m}\right)$, we search for array manifold vectors $\vec{a}(\vec{k})$ which have minimum projection in the noise subspace. We do this by finding peaks in the directional function

$$
D(\vec{k})=\frac{1}{\left|\vec{a}(\vec{k}) \cdot \mathbf{E}_{\mathbf{n}}\right|^{2}}
$$

While equivalent to finding an array manifold vector that lies almost entirely in the signal subspace, finding the minimum projection onto the noise subspace leads to a more precise measurement of the incident signal's direction of arrival.

To find the $q$ signal amplitudes equate USU ${ }^{\dagger}$ with the signal eigenvector covariances

$$
\mathbf{U S U}^{\dagger}=\mathbf{E}_{\mathbf{8}} \mathbf{\Lambda}_{\mathbf{8}} \mathbf{E}_{\mathbf{g}}^{\dagger} \text {. }
$$

Approximating the $q$ signal direction vectors, $\vec{u}\left(\vec{k}_{m}\right)$, by the $q$ array manifold vectors, $\vec{a}(\vec{k})$, which have minimum projection in the noise subspace and inverting for $\mathbf{S}$ we have

$$
\hat{\mathbf{S}}=\left[\mathbf{A}^{\dagger} \mathbf{A}\right]^{-\mathbf{1}} \mathbf{A}^{\dagger} \mathbf{E}_{\mathbf{g}} \mathbf{A}_{\mathbf{B}} \mathbf{E}_{\mathbf{B}}^{\dagger} \mathbf{A}\left[\mathbf{A}^{\dagger} \mathbf{A}\right]^{-\mathbf{1}},
$$

where

$$
\mathbf{A}=\left(\begin{array}{cccc}
\vec{a}\left(\vec{k}_{1}\right) & \vec{a}\left(\vec{k}_{2}\right) & \ldots & \vec{a}\left(\vec{k}_{q}\right) \\
\downarrow & \downarrow & \ldots & \downarrow
\end{array}\right)
$$

The diagonal elements of $\hat{\mathbf{S}}$ are estimates of the signal intensities.

The method described thus far was developed under the assumption that the signals are stationary. When nonstationary signals are incident, the covariances can no longer be written as in Eq. (3). Instead the covariances are

$$
R_{i j}=\sum_{m=1}^{q}\left|A_{m}\right|^{2} e^{i \vec{k}_{m} \cdot\left(\vec{x}_{i}-\vec{x}_{j}\right)}+\sigma^{2} \delta_{i j}+
$$

$$
\left\langle\sum_{m=1}^{q} \sum_{n=1}^{q} A_{m} A_{n} e^{i\left(\vec{k}_{m} \cdot \vec{x}_{i}-\vec{k}_{n} \cdot \vec{x}_{j}+\phi_{m}(t)-\phi_{n}(t)\right)}\right\rangle_{t} .
$$

The first two terms are the same as the stationary covariances of Eq. (3). The last term arises from the interference of the $q$ nonstationary signals. If the MUSIC method were applied directly to these covariances, the number of eigenvalues greater than $\lambda_{\min }$ would be less than the actual number of signals, and thus we could not find the signal direction vectors $\vec{u}(\vec{k})$.

We modified MUSIC to avoid this problem by reducing the contribution of the nonstationary terms in the covariances by averaging the covariances over subarrays. We choose the size of the subarray by combining an information theoretic criteria [Wax and Kalaith, 1985] to estimate the number of signals, with a spatial averaging technique [Shan et al., 1985] to average over subarrays. The size of the subarray is increased incrementally, from a single station, until the number of large eigenvalues of the averaged covariance matrix stops increasing, or until the number of large eigenvalues is equal to $N_{a v g}-1$, where $N_{a v g}$ is the dimension of the averaged covariances. Once the appropriate size of the subarrays has been determined, MUSIC is used as before to find the signal direction vectors $\vec{u}(\vec{k})$ and the signal intensities matrix $\hat{\mathbf{S}}$. In this case the diagonal elements of $\hat{\mathbf{S}}$ are signal intensities and the off diagonal elements are a measure of signal correlation.

\section{Experiments and Results}

In this section we describe a series of experiments that illustrate the capabilities of our algorithm and compare them with Capon's [1969] high resolution method. In each experiment we have used a sevencomponent linear array with equal spacing in a homogeneous medium. The covariance matrix was calculated from synthetic time series which were generated as a linear superposition of stationary and nonstationary plane waves in a background of white noise.

In the first set of experiments we input a single signal in a background of white noise. For both MUSIC and Capon's high resolution method we found that the minimum resolvable signal to noise amplitude ratio was approximately $0.3(\approx-10 \mathrm{~dB})$ independent of angle of arrival or signal correlation. The second set of experiments compared the capabilities of both methods to resolve two, closely-spaced signals. We looked at pairs of either stationary or nonstationary signals in white noise. In Figure 1 we plot the minimum resolvable angular separation versus signal to noise ratio for MUSIC and Capon's method. For stationary signals MUSIC has approximately two and one half times greater resolution than Capon's method. When nonstationary signals are incident, MUSIC's resolution is degraded because the necessary spatial averaging decreases the effective number of sensors N. However, Capon's method cannot resolve nonstationary signals.

In another set of experiments we compared the capabilities of these methods to resolve a large number of signals. In Figure 2 we compare the power spectra of these methods when five stationary signals with closely spaced directions of arrival and varying ampli- 
tude are incident. MUSIC accurately located all five signals. Capon's method was unable to resolve the closely spaced signals but did give a rough estimate of the peak power. An example with four signals three of which are nonstationary is shown in Figure 3. MUSIC resolves all four signals; Capon's method resolves only the stationary signal.

\section{Summary}

We have presented the MUSIC method as a means for analyzing seismic signals recorded at an array of seismometers. The numerical algorithm that implements MUSIC was used to measure directions of arrival and amplitudes of multiple stationary and nonstationary plane waves in a homogeneous medium. In comparisons with Capon's high resolution maximum likelihood method we found that both methods had similar resolution capabilities when a single signal was present. However, when multiple signals were present, some of which were nonstationary, MUSIC had much higher resolution. Further, when the number of signals was larger than the number of sensors, MUSIC was most sensitive to the strongest signals. Of particular importance is the adaptation of MUSIC to deal with nonstationary signals which Capon's method cannot resolve. In principle MUSIC can be used to study nonplanar, broadband, polarized signals propagating in an inhomogeneous medium. Development of numerical algorithms dealing with these conditions is in progress.

Acknowledgements. We would like to thank Leroy Dorman who suggested we look at the MUSIC approach. We would also like to thank Norman Abrahamson, Paul Spudich and an anonymous reviewer for their comments. This work was partially suported by the Office of Nuclear Regulatory Research, U.S. Nuclear Regulatory Commission, Grant NRC-G-04-85-004.

\section{References}

Abrahamson, N.A., Estimation of seismic wave coherency and rupture velocity using the SMART-1 strong motion array recordings, Ph.D. Dissertation, 126 pp., University of California, Berkeley, 1985.

Aki, K., C. Anders and E.S. Husebye, Three dimensional seismic structure of the lithosphere under Montana LASA, Bull. Seismol. Soc. Am. , 64, 637-656, 1976.

Capon, J., R.J. Greenfield and R.J. Kolker, Multidimensional maximum- likelihood processing of a large aperture seismic array, Proc. IEEE , 55, 192-211, 1967.
Capon, J., High resolution frequency-wavenumber spectrum analysis, Proc. IEEE , 57, 1408-1418, 1969.

Capon, J., Characterization of crust and upper mantle structure under LASA as a random medium, Bull. Seismol. Soc. Am. , 64, 235-266, 1974.

Filson, J., Array seismology, Ann. Rev. Earth Planet. Sci. , 3, 157-181, 1975.

Havskov, J. and E.R. Kanasewich, Determination of the dip and strike of the Moho from array analysis, Bull. Seismol. Soc. Am. , 68, 1415-1419, 1978.

Haykin, S. (Ed.), Radar array processing for angle of arrival estimation, In Array Signal Processing, Edited by S. Haykin, Prentice-Hall, Englewood Cliffs, New Jersey, 1985.

Kay, S.M. and S.L. Marple, Jr., Spectrum analysis-a modern perspective, Proc. IEEE , 69, 1380-1418, 1981.

McLaughlin, K.L., L. Johnson and T. McEvilly, Two-dimensional array measurements of near source ground accelerations, Bull. Seismol. Soc. Am. , 73, 349-375, 1983.

Ringdal, F. and E.S. Husebye, Application of arrays in the detection, location, and identification of seismic events, Bull. Seismol. Soc. Am. , 72B, S201-S204, 1982.

Schmidt, R.O., A signal subspace approach to multiple emitter location and spectral estimation, Ph.D. Dissertation, 201 pp., Stanford University, Stanford, California, 1981.

Schmidt, R.O., Multiple emitter location and signal parameter estimation, IEEE Trans. on Antennas and Propagation , 34, 276-280, 1986.

Shan, T.J., M. Wax and T. Kailath, On spatial smoothing for direction of arrival estimation of coherent signals, IEEE Trans. on Acoust., Speech, Signal Process. , 33, 806-811, 1985.

Spudich, P. and E. Cranswick, Direct observation of rupture propagation during the 1979 Imperial Valley, California, earthquake using a short-baseline accelerometer array, Bull. Seismol. Soc. Am. , 74, 2083-2114, 1984.

Wax, M. and T. Kailath, Detection of signals by information theoretic criteria, IEEE Trans. on Acoust., Speech, Signal Process. , 33, 387-392, 1985.

R. J. Archuleta, P. Goldstein, Department of Geological Sciences, University of California, Santa Barbara CA 93106.

(Received October 16, 1986; accepted November 7, 1986.) 